\title{
Caryotypes de Neocanonopsis dreuxi Hoffmann et Christensenia antarctica Brinck, Curculionidae Ectemnorrhininae (Insectes, Coléoptères) de l'archipel Crozet
}

\author{
S Bailly ${ }^{1}, \mathrm{P}$ Dreux ${ }^{2}$ \\ 1 Université $P$ et $M$ Curie (Paris VI), laboratoire de biologie animale, bât $C$, \\ 4 place Jussieu; \\ ${ }^{2}$ École normale supérieure, laboratoire de zoologie, 46 rue d'Ulm, 75230 \\ Paris Cedex 05, France
}

(Reçu le 21 novembre 1991; accepté le 28 mars 1992)

\begin{abstract}
Résumé - Les Ectemnorrhininae (Coléoptères, Curculionides) constituent une sous-famille très originale de Charançons strictement localisés dans les îles du Sud de l'océan Indien, terres de nature volcanique très isolées les unes des autres et, pour certaines, peuplées depuis peu de temps. Ils y constituent le groupe d'Insectes le plus important et proviennent d'une souche primitive d'origine encore inconnue. On en a à ce jour répertorié une trentaine d'espèces, à l'endémisme très fort, dans les archipels et même dans les îles. Leur systématique se fait d'ordinaire à partir de données morphologiques fournies par les adultes ou les larves, ou encore à partir du régime alimentaire. La présente étude entre dans le cadre de travaux qui visent à la connaissance de la phylogénie de ces Curculionides en utilisant une analyse cytogénétique encore jamais réalisée. Dans cette perspective, les caryotypes de 2 genres voisins de la tribu des Canonopsini, Christensenia antarctica et Neocanonopsis dreuxi, ont été établis grâce à un programme d'analyse de données capable d'agréger des objets (ici les chromosomes) caractérisés par un certain nombre d'attributs quantitatifs (ici la longueur et l'indice centromérique) en fonction de leurs similitudes. Dans les 2 espèces, la formule chromosomique a été trouvée du type $10 \mathrm{AA}+\mathrm{X} y_{p}$, commun à plusieurs espèces de Curculionides. Les chromosomes de $N$ dreuxi présentent plus de variations dans les valeurs de l'indice centromérique que ceux de $C$ antarctica. Les résultats sont rapprochés de ceux obtenus sur 3 Ectemnorrhinini lors d'une étude préliminaire. L'analyse d'une plus large gamme d'espèces et de genres, prévue par les auteurs, demeure cependant nécessaire à toute conclusion phylogénétique.
\end{abstract}

Coléoptère, Curculionide / Ectemnorrhininae / îles Crozet / cytogénétique / caryotype 
Summary - Karyotypes of Neocanonopsis dreuxi Hoffmann and Christensenia antarctica Brinck, Curculionidae Ectemnorrhininae (insects, Coleoptera) from Crozet Archipelago. Ectemnorrhininae (Coleoptera, Curculionidae) represent a specific subfamily of weevils, which are found only on volcanic islands in the Southern Indian ocean. The islands, which are largely isolated from each other, constitute a distinctly individualized biogeographical province. On this archipelago, Ectemnorrhininae form the most important group of insects, originating from a primitive stock, the origin of which although still unknown, is probably antarctic. This group includes some 30 recorded species, which appear to be differentially distributed among the islands, some of these islands having been only recently inhabited owing to the recent period of their emergence. Until now, classification of Ectemnorrhininae was essentially established on the sole basis of adult and larval morphological criteria. More recently, alimentary habits have been taken into account. The present study is part of a more comprehensive approach, aimed at understanding the Curculionidae phylogeny, using cytogenetic analysis, which has not been done before. Karyotypes of 2 closely related species pertaining to the Canonopsini tribe, Christensenia antarctica and Neocanonopsis dreuxi, were established, utilizing a computerized analysis which allows the grouping of objects (here chromosomes) in accordance with similarities (here length and centromeric index) detected between them. Both species are characterized by the same general chromosomal formula, $10 A A+X y_{p}$, this type of formula being shared by many species of Curculionidae. Centromere location is more variable in chromosomes of $\mathrm{N}$ dreuxi than in those of $\mathrm{C}$ antarctica. These observations are compared with the findings of a previous study which determined the karyotype of 3 different species of Ectemnorrhinini. Further analytical studies using a wider range of genera and species are required for establishing complete phylogenetic relationships.

Coleoptera, Curculionidae / Ectemnorrhininae / Crozet archipelago / cytogenetics / karyotype

\section{INTRODUCTION}

Les Ectemnorrhininae (Coléoptères, Curculionides) constituent une sous-famille très originale de charançons strictement localisés dans les îles du Sud de l'océan Indien : îles Marion et du Prince Edouard, archipel Crozet, archipel de Kerguelen, îles Macdonald et Heard (Dreux et Voisin, 1987). Ces terres d'origine volcanique, isolées les unes des autres ainsi que des continents les plus proches, forment une province biogéographique bien individualisée, à flore et faune pauvres, avec les Ectemnorrhininae comme groupe d'Insectes le plus important. L'origine de leur souche primitive, inconnue, est supposée sur le continent antarctique. Tous ont une reproduction sexuée biparentale.

Ce groupe comprend actuellement, réparties en une dizaine de genres, une trentaine d'espèces dont beaucoup sont endémiques d'un des archipels et même d'une seule île. L'histoire de l'évolution du groupe, peuplant des îles volcaniques d'apparition récente, est donc d'un très grand intérêt à cause des nombreuses spéciations qui s'y sont produites, certaines probablement depuis peu de temps.

Jusqu'à présent, la systématique des Ectemnorrhininae a été presqu'exclusivement fondée sur la morphologie des adultes, la première étude importante étant due à Jeannel (1940). Dans une mise au point récente, Dreux et Voisin (1989) rappellent l'essentiel de la bibliographie et présentent un essai de reconstitution de l'arbre 
phylogénique. La morphologie larvaire (Chown et Scholtz, 1989) et le régime alimentaire (Chown, 1989) commencent à apporter de nouveaux éléments mais ceux-ci demeurent encore fragmentaires.

L'examen des caryotypes, non entrepris auparavant, semble être une autre voie d'approche du problème. C'est pourquoi, pour commencer une telle étude, une première campagne de prélèvements d'échantillons a eu lieu pendent l'été austral 1988-1989 et a permis la collecte par l'un de nous (P Dreux) d'une douzaine d'espèces bien réparties parmi les genres puisque ceux-ci, à l'exception de 2 seulement, sont tous représentés. Trois de ces espèces ont déjà été étudiées d'un point de vue cytogénétique (Bailly et al, 1990). Il s'agit d'Ectemnorrhinus viridis et de $E$ drygalskii (de Kerguelen) et de Dusmoecetes richtersi (de l'archipel Crozet), 2 genres d'Ectemnorrhininae voisins, appartenant à la tribu la plus homogène, celle des Ectemnorrhinini.

Le présent travail porte sur 2 autres espèces, Christensenia antarctica Brinck (1945) et Neocanonopsis dreuxi Hoffmann (1966), dont la tribu, les Canonopsini, se compose de 4 genres monospécifiques très différents, ayant seulement en commun un pronotum présentant des creux et des bosses, ainsi que des élytres recouvrant le pygidium (Dreux et Voisin, 1987).

Dans cette étude, le but poursuivi est double : continuation de l'étude caryologique des Ectemnorrhininae et, partant, meilleure connaissance de leur phylogénie.

\section{MATÉRIEL ET MÉTHODES}

\section{Zone d'étude et capture}

Les espèces étudiées sont de grande taille, $C$ antarctica pouvant atteindre jusqu'à $2 \mathrm{~cm}$. Elles se trouvent en nombre sous les pierres du «fell-field», étendue de cailloux située en altitude et présentant une végétation très clairsemée. Ces Curculionides sont répandus dans toutes les îles de l'Archipel à l'exception de l'île aux Cochons, la plus récemment émergée (moins de $10^{6}$ ans) où $N$ dreuxi n'a encore jamais été observé.

Les échantillons utilisés ici ont été récoltés par l'un de nous (P Dreux) durant l'été austral à l'île de la Possession, au lieu dit «le Plateau des Pétrels» (altitude $600 \mathrm{~m}$ ) et malgré une neige récemment tombée. Les Curculionides ont ensuite été conservés vivants à bord du Marion-Dufresne, navire ravitailleur des Terres Australes et Antarctiques Françaises, dans des boîtes en plastique garnies d'un peu de végétation et maintenues au froid (entre 0 et $4^{\circ} \mathrm{C}$ ) jusqu'à l'arrivée au laboratoire de biologie de Kerguelen.

\section{Prélèvement des testicules et fixation}

Ces opérations se déroulent au laboratoire de Kerguelen. La dissection s'effectue sur l'animal frais, immobilisé à la surface d'une plaque poreuse dans du liquide de Ringer. Le sexe mâle est indiqué par la présence du pénis, long et très sclérifié, dans la moitié postérieure de la cavité abdominale. En effet, si $N$ dreuxi présente un dimorphisme sexuel externe, le sexe de $C$ antarctica n'apparaît qu'à la dissection. Les testicules sont des masses blanchâtres formées d'une quinzaine de sphérules, 
situées à peu près à la hauteur de l'avant du pénis. Des spermiductes bien visibles les relient à la base de celui-ci. Prélevés à l'aide d'une paire de pinces fines, les testicules sont placés pendant 20 min dans une solution hypotonique de $\mathrm{NaCl}(1,7 \mathrm{~g} / \mathrm{l})$ puis plongés $40 \mathrm{~min}$ dans du fixateur de Carnoy (3/1). On les lave ensuite dans de l'alcool absolu en 3 bains successifs de 20 min chacun. La conservation se fait dans des microtubes remplis de butanol, à raison d'une seule paire de testicules par microtube.

\section{Techniques cytologiques}

Les testicules sont à nouveau rincés dans l'alcool absolu à raison de 3 bains successifs de $10 \mathrm{~min}$ chacun. Ils séjournent ensuite 2 fois $7 \mathrm{~min}$ dans des bains différents d'acide acétique (50\%). On procède alors à l'étalement des chromosomes sur lames histologiques selon la technique du choc thermique (Bailly, 1982). Après 2 rinçages de $2 \mathrm{~min}$ chacun dans du Carnoy (3/1), les préparations restent ensuite $25 \mathrm{~min}$ dans un bain colorant d'orcéine acétique (1\%). Le montage se fait dans de l'Eukitt après 2 passages successifs dans l'alcool absolu et le toluène.

\section{Observation des préparations}

Un microscope Zeiss Universal équipé d'un objectif 100 à contraste de phase permet l'observation des préparations ainsi que la prise de photographies sur lesquelles sont ensuite relevées les mensurations des chromosomes.

\section{Analyse statistique des résultats}

L'établissement d'un caryotype consiste en un rangement des chromosomes par paires en fonction de critères (indice centromérique et longueur) caractéristiques de ces dernières. Si les critères ne sont pas connus, comme c'est le cas pour le matériel étudié ici, il convient de les déterminer avant toute tentative d'établissement de caryotype, d'autant plus que les chromosomes des 2 espèces de Charançons apparaissent morphologiquement très uniformes. Le processus choisi consiste à traiter l'ensemble de tous les chromosomes mesurés, pour une espèce donnée, de manière à les regrouper en «classes » les plus homogènes possible correspondant aux différentes paires chromosomiques, le classement devant s'effectuer en fonction de l'indice centromérique et de la longueur. Il suffit ensuite de déterminer, pour chaque classe, les valeurs moyennes d'indice et de longueur pour connaître du même coup les valeurs caractérisant les chromosomes de chacune des paires. Quand ces valeurs sont connues, on peut alors construire le caryotype de manière rigoureuse.

Pour ce faire, on a fait appel, dans ce travail, au programme d'analyse de données CONSTEL (Meyer, 1974). Ce programme est capable d'agréger des objets caractérisés par un certain nombre d'attributs quantitatifs (ici la longueur et l'indice centromérique) en fonction de leurs similitudes. Il est basé sur une description de ces objets à partir d'une analyse en composantes principales du tableau initial de données. Ce dernier inclut chaque observation concernant l'objet étudié (ici le chromosome) et les variables qui le déterminent. Ce programme utilise une méthode de regroupement des classes décrite antérieurement par Carlson (1972). L'algorithme repose sur une recherche du plus proche voisin, limitée par un critère 
statistique visant à intégrer un nouvel objet dans une classe donnée seulement si sa distance aux objets de la classe est statistiquement plus faible que sa distance à tout objet extérieur à la classe.

L'indice centromérique $(i)$ est défini dans ce travail comme égal à 100 fois le rapport de la longueur du bras court à la longueur totale du chromosome (Levan et $a l, 1964)$. La longueur du chromosome est exprimée en longueur relative (LR), soit 100 fois le rapport de la longueur du chromosome considéré à celle du «complément» (c'est-à-dire la moitié de la longueur totale du lot diploïde de chromosome, X et Y inclus).

\section{RÉSULTATS}

Pour $C$ antarctica, 6 mâles ont été récoltés et les testicules de 5 d'entre eux ont servi à la présente étude. Pour $N$ dreuxi, les chiffres sont respectivement de 10 et de 5 .

Dans les 2 espèces, les préparations montrent de très nombreuses figures de méiose. Le nombre de stades diacinèse et métaphase I où l'on peut compter aisément le nombre de bivalents varie en effet, selon les préparations, de 200 à 320 pour un seul testicule. Les figures de mitoses, qui correspondent à des divisions spermatogoniales, sont, en revanche, beaucoup moins fréquentes, voire rares. Toutefois, pour chaque espèce, 13 plaques métaphasiques ont pu être sélectionnées pour servir aux calculs statistiques. Il s'agit de plaques où la bonne disposition des chromosomes permet à ceux-ci d'être aisément comptés et, pour la plupart, mesurés. Pour cette dernière opération, en effet, quelques chromosomes, trop repliés ou entrecroisés, n'ont pu être utilisés, la position du centromère n'étant pas décelable de façon certaine. C'est pourquoi finalement 162 chromosomes mitotiques, fournissant 162 couples de données, ont été utilisés par le programme d'analyse chez $C$ antarctica et 201 chez $N$ dreuxi.

\section{Formule chromosomique}

Les figures 1 et 2 présentent des stades de divisions (méiose et mitose) caractéristiques de $C$ antarctica et de $N$ dreuxi. Dans les 2 espèces, le nombre de bivalents en prophase I de méiose ainsi que le nombre de chromosomes formant les plaques métaphasiques de mitose conduisent à la valeur diploïde $2 n=22$. Au state de la métaphase I de méiose (figure 1, c et d; figure 2, c et e), les chromosomes sexuels forment la figure dite «en parachute» propre aux Coléoptères (Stevens, 1906), conséquence de l'importante différence de taille existant entre les chromosomes X et Y. Compte tenu de la terminologie en usage (Smith, 1950), la formule chromosomique de ces 2 Curculionides est donc du type $10 \mathrm{AA}+\mathrm{X} y_{p}$. Aucun cas d'aneuploïdie n'a été observé.

\section{Figures de méiose}

Dans les 2 espèces, les chromosomes très ténus et enchevêtrés au stade leptotène (fig 1a et 2a) s'apparient pour former dès le pachytène, des bivalents déjà bien 

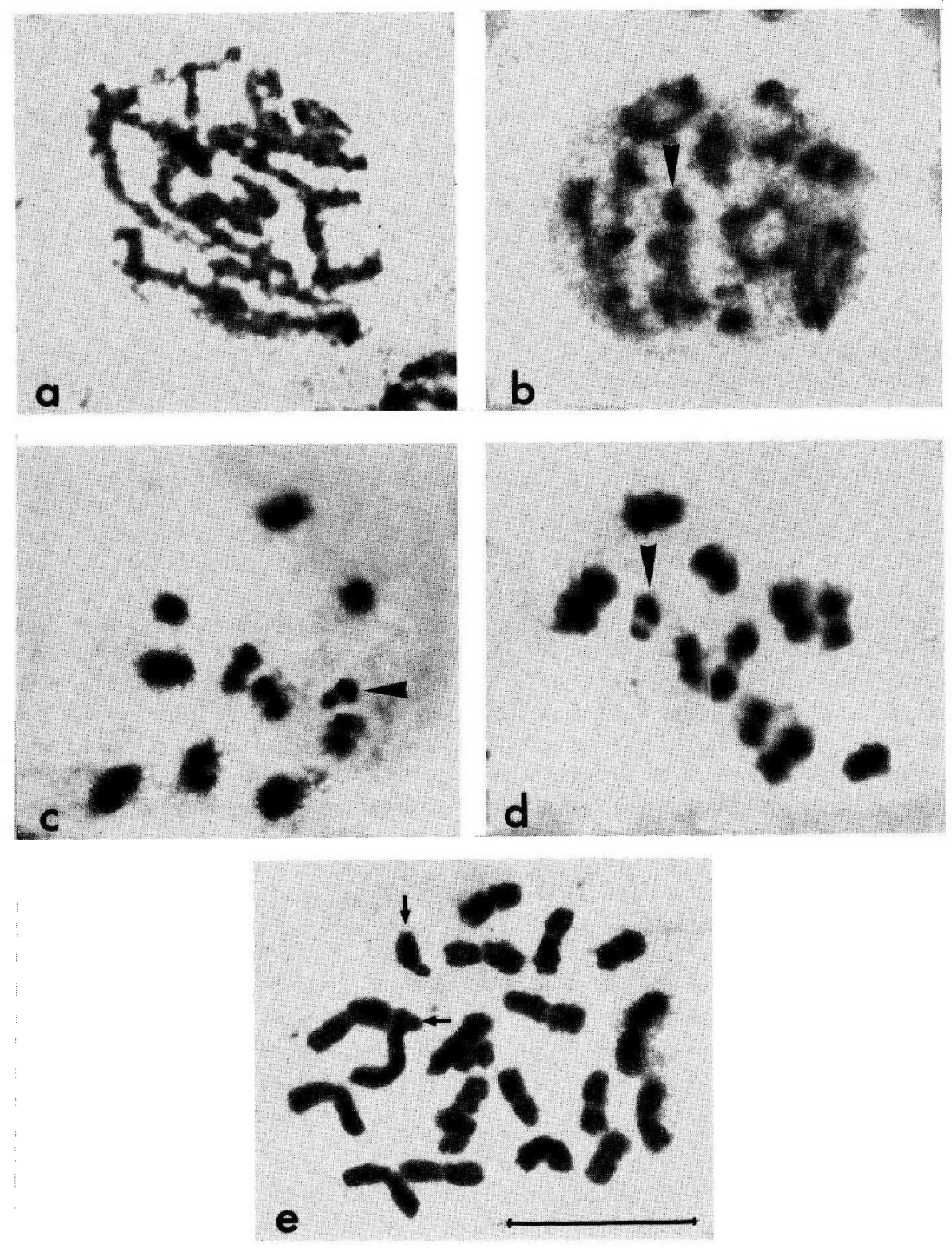

Fig 1. Christensenia antarctica. Figures de méiose : a : stade leptotène; $\mathbf{b}$ : stade diplotène; $\mathbf{c}$ : métaphase 1 , vue polaire. $\mathbf{d}$ : métaphase 1 , vue équatoriale. Les flèches indiquent le bivalent sexuel formant la disposition «en parachute». Figure de mitose : e : métaphase. Les flèches indiquent les chromosomes sexuels X et Y, ce dernier étant le plus petit. La barre représente $10 \mu \mathrm{m}$. 

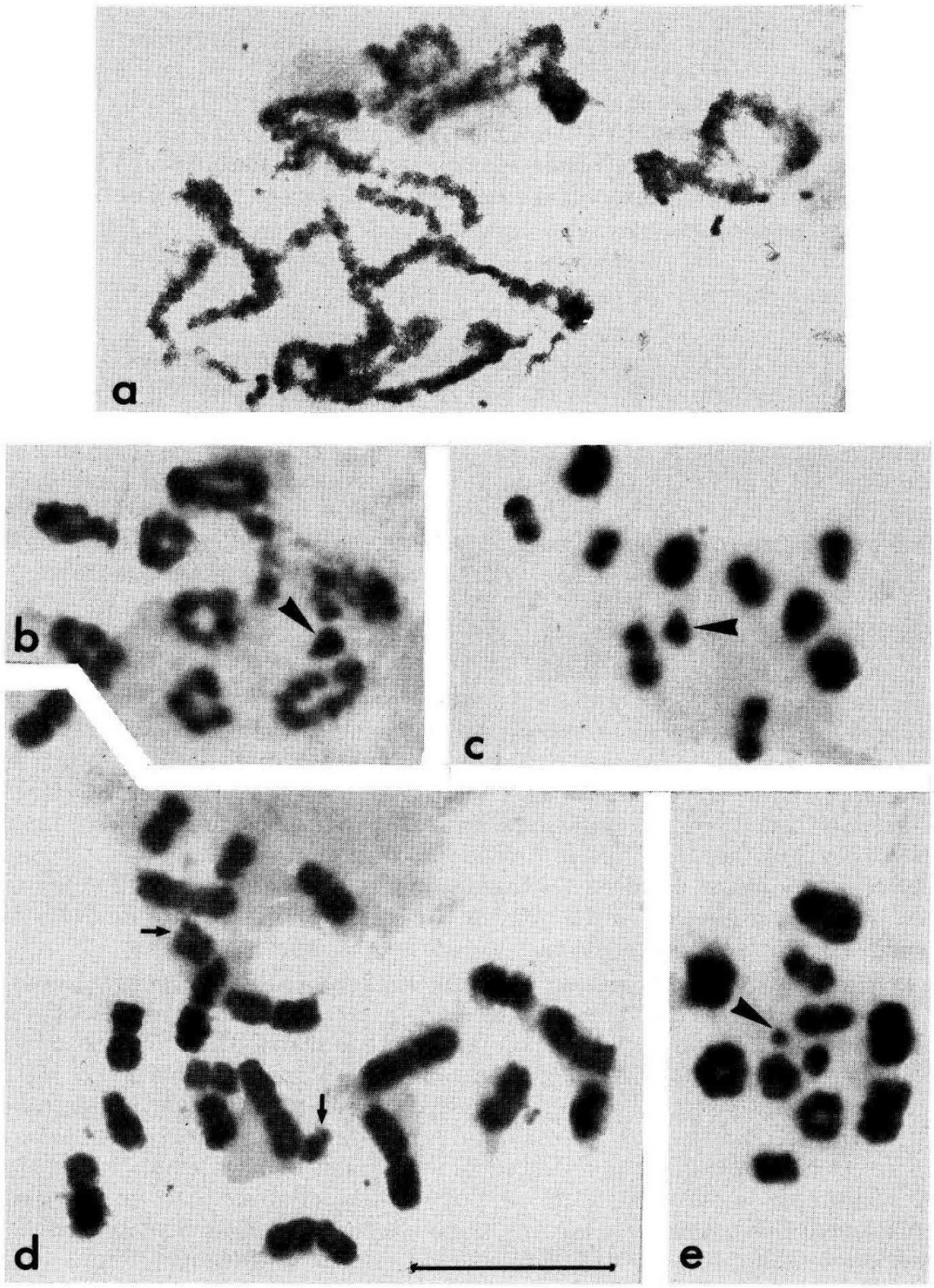

Fig 2. Neocanonopsis dreuxi. Figures de méiose : a : stade leptotène; $\mathbf{b}:$ stade diplotène; c : métaphase 1 , vue équatoriale. $\mathbf{e}:$ métaphase 1 , vue polaire. Les flèches indiquent le bivalent sexuel formé «en parachute». Figure de mitose : $\mathbf{d}$ : plaque métaphasique. Les flèches indiquent les chromosomes sexuels $\mathrm{X}$ et $\mathrm{Y}$, ce dernier étant le plus petit. La barre représente $10 \mu \mathrm{m}$. 
reconnaissables. Toutefois, il n'est pas encore possible d'identifier parmi eux les chromosomes sexuels que ne signale aucune hétéropycnose. En revanche, au stade diplotène, tous les bivalents sont bien distincts (fig $1 \mathrm{~b}$ et $2 \mathrm{~b}$ ), y compris le bivalent sexuel qui présente, dès ce stade, la disposition en parachute. L'emplacement des chiasmas est tout à fait net. Ultérieurement, la condensation atteint son maximum en métaphase I (fig 1c, d; 2c, e). Dans le bivalent sexuel, le chromosome $Y$ se réduit à un point et aucune relation n'existe entre ce bivalent et l'un ou l'autre de ceux formés par les autosomes.

\section{Établissement du caryotype}

Dans les 2 espèces, les chromosomes sexuels sont facilement repérables. Le chromosome $\mathrm{Y}$, souvent ponctiforme, est de loin le plus petit de tous (fig 1e et $2 \mathrm{~d}$ ). Le chromosome X, plus grand, a une taille voisine de celle des petits autosomes mais ne peut être confondu avec aucun d'entre eux à cause de son indice centromérique. Conséquence de leur petite taille et de la contraction souvent importante de leur chromatine qui rend difficile le repérage du centromère, tous les chromosomes $\mathrm{X}$ observés n'ont pu être correctement mesurés. C'est pourquoi 6 d'entre eux seulement, pour chacune des espèces, ont pu servir au calcul des valeurs moyennes (tableaux I et II).

L'ensemble des autosomes ayant fait l'objet de mesures a été, pour chaque espèce, traité par le programme d'analyse CONSTEL. Leur regroupement en classes appelées ici «constellations» apparaît figure 3 pour $C$ antarctica et figure 4 pour $N$ dreuxi. Chacun des carrés y représente au moins un couple de données $(i$ et $L R$ ), c'est-à-dire un chromosome; il peut aussi en représenter plusieurs si plusieurs chromosomes ont les mêmes caractéristiques. Les rectangles sont en fait les produits de la juxtaposition de carrés consécutifs figurant des chromosomes aux coordonnées voisines. L'étoile située à l'intérieur de chaque constellation indique l'emplacement du barycentre de cette dernière. Le programme d'analyse a écarté 12 couples de données pour $C$ antarctica, et 15 pour $N$ dreuxi. Les carrés correspondants sont situés à l'extérieur des constellations.

Le programme d'analyse avait en fait, dans les 2 cas, isolé seulement 9 constellations. Chez $C$ antarctica, les points inclus dans la constellation $\mathrm{n}^{\circ} 10$ étaient réunis à ceux de la constellation $n^{\circ} 7$. Or, si on représente la distribution de l'ensemble de ces points en fonction de la seule longueur, on obtient une courbe à 2 pics séparés (ce qui n'est pas le cas pour les populations de points regroupés par le programme dans les autres constellations). C'est donc pour affiner la répartition initiale des points de cet ensemble que l'on a procédé à l'isolement de la constellation $n^{\circ} 10$, en tenant compte de la distribution de ces points en fonction de leur seule longueur. L'emplacement des barycentres traduit d'ailleurs très bien cette distribution. Chez $N$ dreuxi, les points des constellations 6 et 4 étaient initialement réunis et une analyse identique à celle pratiquée chez $C$ antarctica a conduit à l'isolement de la constellation $\mathrm{n}^{\circ} 6$.

Considérant désormais que chaque constellation correspond au regroupement de points représentant des chromosomes appartenant à la même paire d'autosomes, il suffit de calculer les coordonnées des barycentres des différentes constellations pour connaitre les valeurs moyennes d'indice et de longueur caractérisant les 


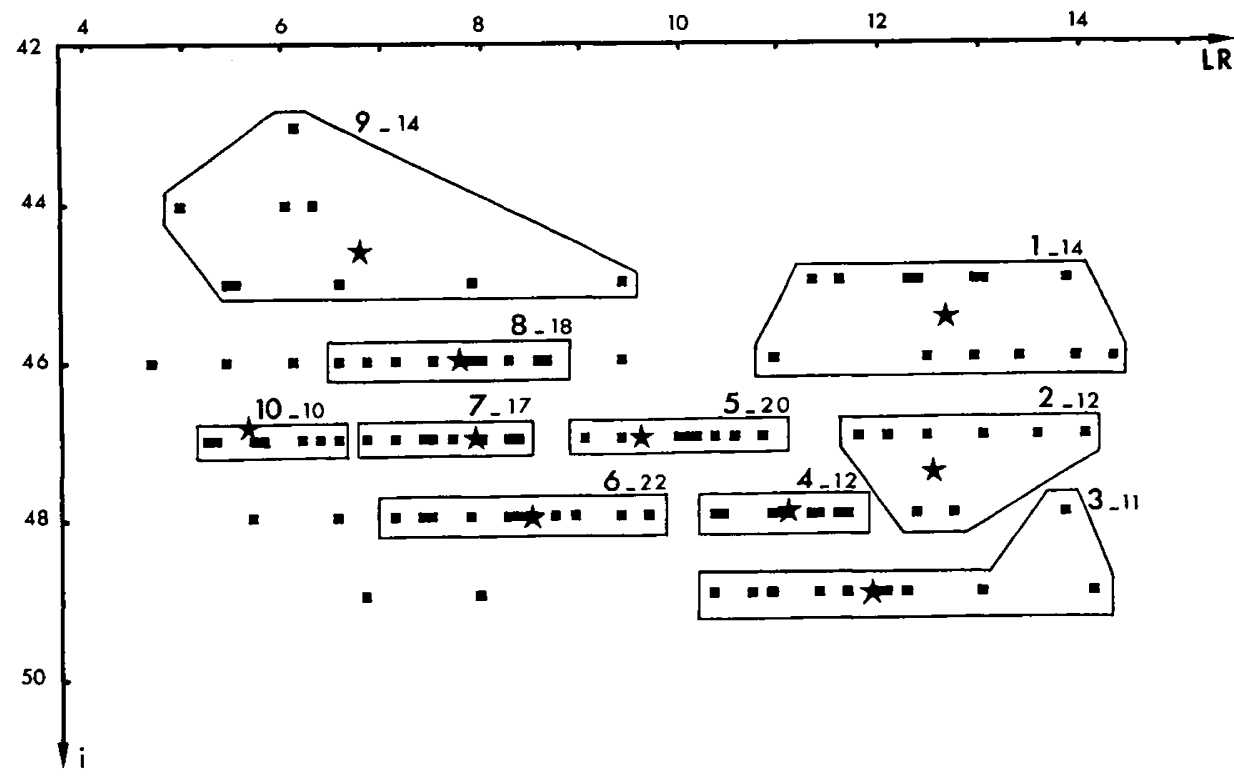

Fig 3. $C$ antarctica. Répartition par traitement statistique de l'ensemble des autosomes déterminés chacun par un couple de données : longueur relative (LR) et indice centromérique (i). Chaque "constellation» regroupe des points correspondant à des chromosomes appartenant à la même paire. Les chiffres indiquent, le premier, le rang de la paire d'autosomes, le second le nombre de couples de données (et donc de chromosomes) traités. Chaque carré peut représenter un ou plusieurs couples de données identiques. Des carrés contigus constituent les rectangles. Les carrés hors des constellations représentent les couples de données écartés par le programme d'analyse. Les étoiles à l'intérieur de chaque constellation indiquent l'emplacement de leurs barycentres dont les coordonnées figurent tableau I.

chromosomes de chacune des paires. Ces valeurs sont rassemblées tableaux I et II où figurent également les écart types des moyennes. Une représentation graphique du caryotype standard peut en être tirée (fig 5). Le système de références étant maintenant entièrement connu, il est désormais possible de dresser un caryotype à partir d'une plaque métaphasique donnée. C'est ce qui a été fait figure 6 à partir des plaques métaphasiques présentées figures 1e et $2 \mathrm{~d}$.

Si l'on s'en tient à la classification de Levan et al (1964), on constate que les autosomes des 2 espèces appartiemnent tous à la catégorie métacentrique («m») puisque leur indice $i$ est supérieur à 37,5. Il en est de même pour le chromosome $\mathrm{X}$ de $C$ antarctica tandis que le chromosome $\mathrm{X}$ de $N$ dreuxi est submétacentrique («sm») avec $i=35,17$. 


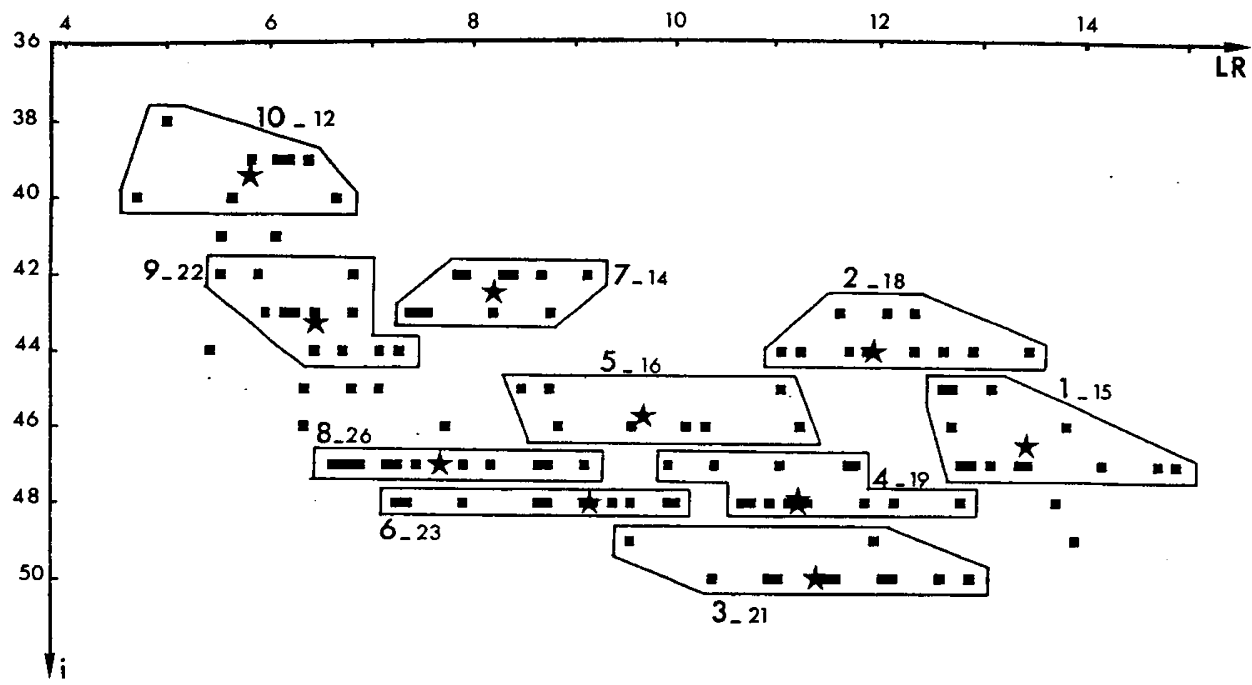

Fig 4. $N$ dreuxi. Répartition par traitement statistique de l'ensemble des autosomes déterminés chacun par un couple de données : longueur relative $(L R)$ et indice centromérique $(i)$. Le regroupement des points par «constellation » est traité de la même manière et avec les mêmes conventions que pour la figure 3 . Les étoiles indiquant l'emplacement des barycentres ont pour coordonnées les couples de valeurs figurant tableau III.

Tableau I. C antarctica : chromosomes mitotiques. Coordonnées moyennes caractérisant chacun des chromosomes des 10 paires d'autosomes ( $n^{\text {os }} 1$ à 10) et le chromosome X.

\begin{tabular}{lccccc}
\hline $\begin{array}{l}\text { Numéro des } \\
\text { paires de } \\
\text { chromosomes }\end{array}$ & $\begin{array}{c}\text { Nombre de } \\
\text { chromosomes } \\
\text { traités }\end{array}$ & $\begin{array}{c}\text { Longueur } \\
\text { moyenne } \\
(L R)\end{array}$ & $\begin{array}{c}\text { Écart } \\
\text { type }\end{array}$ & $\begin{array}{c}\text { Indice } \\
\text { centromérique } \\
\text { moyen }(i)\end{array}$ & $\begin{array}{c}\text { Écart } \\
\text { type }\end{array}$ \\
\hline 1 & 14 & 12,58 & 0,265 & 45,43 & 0,148 \\
2 & 12 & 12,50 & 0,193 & 47,42 & 0,150 \\
3 & 11 & 11,95 & 0,364 & 48,91 & 0,095 \\
4 & 12 & 10,99 & 0,148 & 48,00 & 0,000 \\
5 & 20 & 9,66 & 0,113 & 47,00 & 0,000 \\
6 & 22 & 8,44 & 0,169 & 48,00 & 0,000 \\
7 & 17 & 7,86 & 0,130 & 47,00 & 0,000 \\
8 & 18 & 7,76 & 0,150 & 46,00 & 0,000 \\
9 & 14 & 6,78 & 0,441 & 44,58 & 0,018 \\
10 & 10 & 5,77 & 0,137 & 46,75 & 0,150 \\
$\mathrm{X}$ & 6 & 5,84 & 0,254 & 39,5 & 0,427 \\
\hline
\end{tabular}

Les numéros des paires chromosomiques renvoient aux numéros des constellations de la figure 3 et le nombre de chromosomes indiqué au nombre de couples de données traités, soit le nombre de points inclus dans chaque constellation. 
Tableau II. $N$ dreuxi : chromosomes mitotiques. Coordonnées moyennes caractérisant chacun des chromosomes des 10 paires d'autosomes $\left(n^{\text {os }} 1-10\right)$ et le chromosome X.

\begin{tabular}{lccccc}
\hline $\begin{array}{l}\text { Numéro des } \\
\text { paires de } \\
\text { chromosomes }\end{array}$ & $\begin{array}{c}\text { Nombre de } \\
\text { chromosomes } \\
\text { traités }\end{array}$ & $\begin{array}{c}\text { Longueur } \\
\text { moyenne } \\
(\text { LR) }\end{array}$ & $\begin{array}{c}\text { Écart } \\
\text { type }\end{array}$ & $\begin{array}{c}\text { Indice } \\
\text { centromérique } \\
\text { moyenne (i) }\end{array}$ & $\begin{array}{c}\text { Écart } \\
\text { type }\end{array}$ \\
\hline 1 & 15 & 13,55 & 0,220 & 46,34 & 0,239 \\
2 & 18 & 12,04 & 0,153 & 43,83 & 0,099 \\
3 & 21 & 11,52 & 0,212 & 49,85 & 0,084 \\
4 & 19 & 11,27 & 0,178 & 47,74 & 0,108 \\
5 & 16 & 9,66 & 0,249 & 45,69 & 0,129 \\
6 & 23 & 9,12 & 0,178 & 48,00 & 0,000 \\
7 & 14 & 8,07 & 0,167 & 42,43 & 0,148 \\
8 & 26 & 7,61 & 0,251 & 47,00 & 0,000 \\
9 & 22 & 6,26 & 0,143 & 43,19 & 0,174 \\
10 & 12 & 5,59 & 0,195 & 39,25 & 0,194 \\
$\mathrm{X}$ & 6 & 5,26 & 0,093 & 35,17 & 0,605 \\
\hline
\end{tabular}

Les numéros des paires chromosomiques renvoient aux numéros des constellations de la figure 4 et le nombre de chromosomes indiqué au nombre de couples de données traités, soit le nombre de points inclus dans chaque constellation.
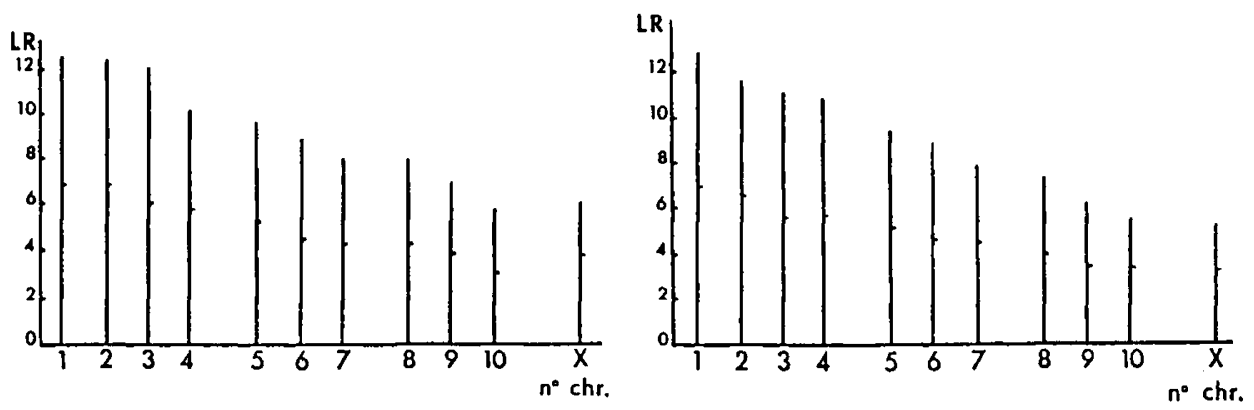

Fig 5. Figuration graphique des chromosomes de $C$ antarctica (A) et de $N$ dreuxi (B). Un autosome par paire $\left(\mathrm{n}^{\circ \mathrm{S}} 1-10\right)$ a été représenté, à partir des données des tableaux I et II. Le chromosome sexuel X seul est indiqué, Y étant le plus souvent ponctiforme. Sur chaque chromosome, l'emplacement du centromère est signalé par un point. La longueur est indiquée en longueur relative $(L R)$.

\section{DISCUSSION}

Si l'on se réfère aux résultats rapportés par Takenouchi en 1984 et replacés par l'auteur dans un ensemble d'analyses chromosomiques portant sur un grand nombre 

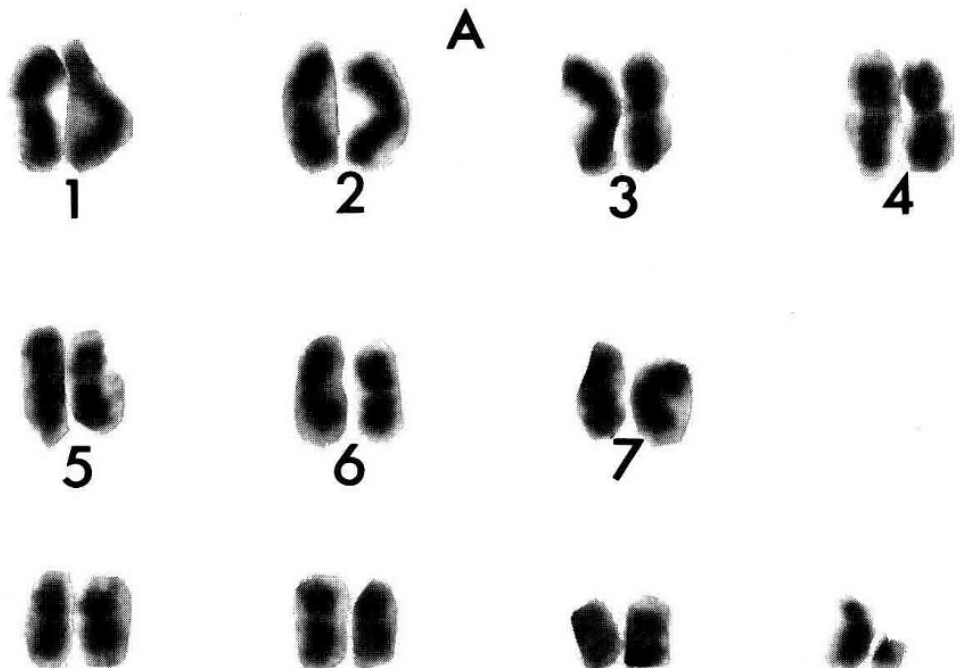

8
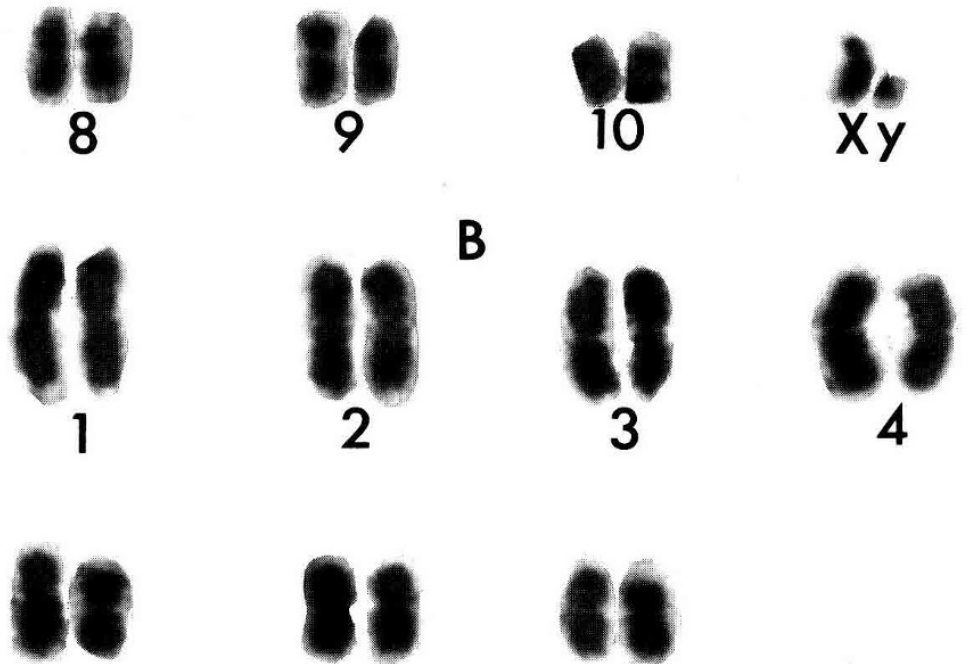

5

6

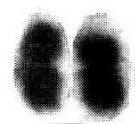

7
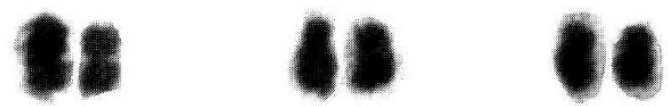

8

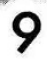

10

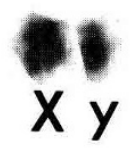

Fig 6. Caryotypes de $C$ antarctica (A) et de $N$ dreuxi (B). Les paires d'autosomes sont numérotées de 1 à 10 . $\mathrm{X}$ et y désignent les chromosomes sexuels. La barre représente $10 \mu \mathrm{m}$. Ces chromosomes sont ceux des plaques métaphasiques des figures 1 , e et 2 , d respectivement. 
d'espèces de Curculionides bisexués, la formule chromosomique $2 n=22$ (10 AA + $\mathrm{X} y_{p}$ ) relevée chez les 2 Canonopsini étudiés ici se situe exacement dans la moyenne des formules connues. De plus, le bivalent sexuel, sans aucun lien avec les bivalents d'autosomes, est conforme à ce qui semble de règle chez les Curculionides, si l'on excepte certains Heilipodus où des systèmes chromosomiques sexuels plus ou moins compliqués ont été décrits (Postiglioni et al, 1987). De même, la formule X $y_{p}$ est la plus communément rencontrée : en 1986, Dey mentionne- seulement 9 espèces de Curculionides où le mécanisme de détermination du sexe est d'un autre type $\left(\mathrm{X} y y_{p}\right)$. Par ailleurs, chez $C$ antarctica et $N$ dreuxi, les centromères des autosomes mitotiques sont nets, facilement identifiables, ce qui n'est pas toujours le cas chez les Charançons : en 1988, Barrion et al décrivent en effet chez plusieurs Sitophilus des centromères de type diffus. Enfin, les autosomes examinés ici appartiennent à la catégorie métacentriques «m» de Levan et al (1964), configuration déjà relevée ailleurs, par exemple chez certains Phyllobiini (Otiorrhynchinae) par Virkki et Sepúlveda en 1990.

En comparant entre elles les observations faites chez $C$ antarctica et $N$ dreuxi, on peut mettre en évidence quelques différences intéressantes. Chez les autosomes, elles apparaissent surtout à propos des indices centromériques (fig 5 , tableaux I et II). Chez $C$ antarctica, il y a peu de variations d'un autosome à l'autre : en effet, à une exception près (chromosome 9, indice 44,5), tous les indices sont compris entre 46 et 49 . Chez $N$ dreuxi, au contraire, les écarts sont plus importants puisque compris entre 39 et 50, avec 4 autosomes pour lesquels l'indice est inférieur à 46 . Même tendance pour le chromosome $\mathrm{X}: i=39,5$ chez $C$ antarctica et 35,17 chez $N$ dreuxi. Seconde différence importante, le chromosome $\mathrm{X}$ de $C$ antarctica est un peu plus grand que le plus petit autosome. C'est le contraire chez $N$ dreuxi. En conséquence, on peut considérer les caryotypes de $C$ antarctica et $N$ dreuxi comme très proches, avec plus d'asymétrie dans la position'du centromère chez ce dernier.

Il peut être intéressant de comparer ces résultats avec ceux enregistrés sur 3 autres Ectemnorrhininae, Ectemnorrhinus drygalskii, E viridis et Dusmoecetes richtersi, étudiés précédemment (Bailly et al, 1990) qui appartiennent à la tribu des Ectemnorrhinini. Pour ces 3 espèces de même origine géographique, les formules chromosomiques sont identiques : $2 n=22$, de type $10 \mathrm{AA}+\mathrm{X} y_{p}$. Également, en prophase I de méiose, le bivalent sexuel n'est associé à aucun autre bivalent d'autosomes et en métaphase de mitose les autosomes des 3 espèces sont de taille équivalente. En revanche, les relevés d'indices centromériques mettent en évidence une position du centromère plus asymétrique que chez les 2 Cananopsini. En effet, chez $E$ drygalskii, l'indice varie de 50 à 25 ; de 50 à 37 chez $E$ viridis et de 50 à 34 chez $D$ richtersi (fig 7 ).

Après l'étude de 5 espèces seulement, il est un peu tôt pour avancer des hypothèses. Toutefois, on peut remarquer que, chez les 3 Ectemnorrhinini, 2 ont des chromosomes submétacentriques, ce qui n'est pas le cas chez les Canonopsini. Ceci peut apparaitre comme une donnée cytogénétique venant confirmer la distinction établie entre ces 2 tribus sur la seule base de l'examen morphologique. En revanche, il serait prématuré d'en déduire l'existence d'un rapport plus étroit entre $N$ dreuxi et les Ectemnorrhinini. Il convient auparavant d'analyser une plus large gamme d'espèces et de genres, ce qui est l'objectif des auteurs. 


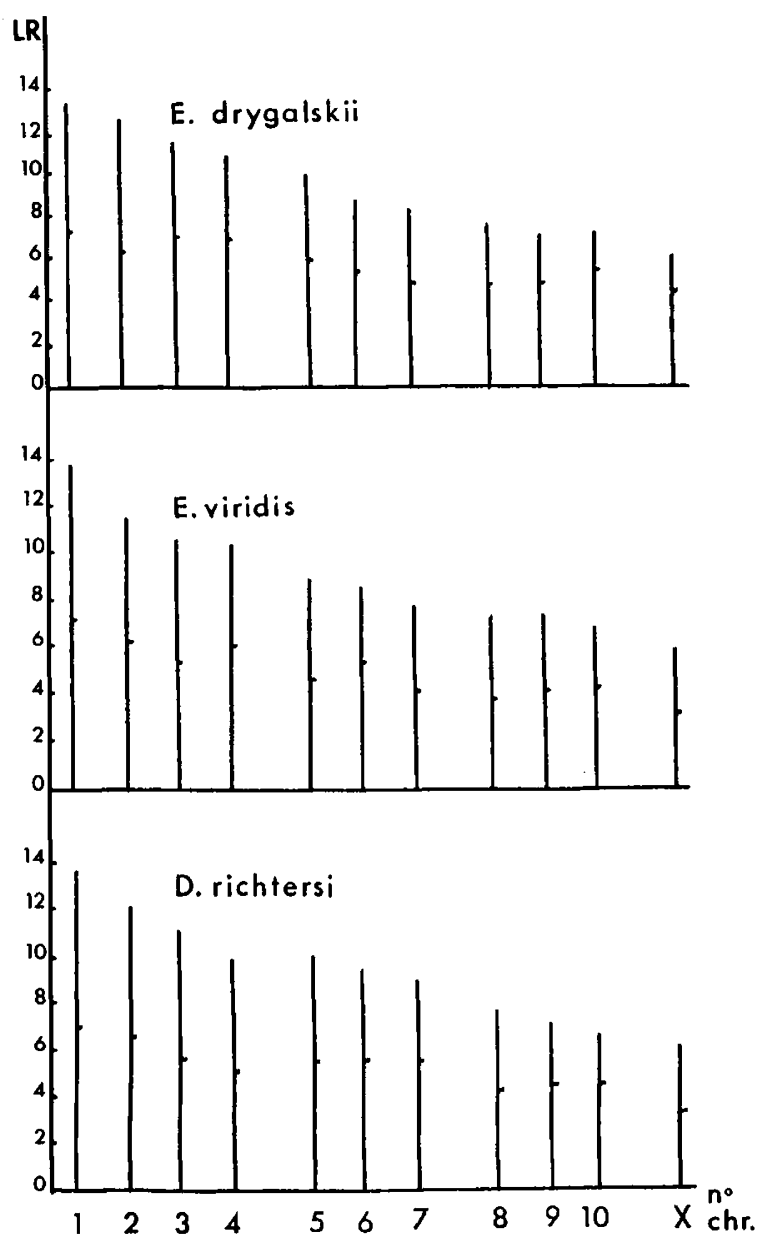

Fig 7. Rappel graphique des résultats d'une étude préliminaire portant sur Ectemnorrhinus drygalskii, E viridis et Dusmoecetes richtersi. Un autosome par paire $\left(\mathrm{n}^{\circ \mathrm{s}} 1-10\right)$ est figuré, ainsi que le chromosome sexuel $\mathrm{X}$; $\mathrm{Y}$, étant ponctiforme, n'est pas représenté. L'emplacement des centromères est signalé par un point. La longueur est indiquée en longueur relative $(L R)$.

\section{REMERCIEMENTS}

Nous remercions l'administration des Terres Australes et Antarctiques Françaises, et en particulier le chef de la Mission de Recherches, B Morlet, d'avoir donné à l'un de nous (P Dreux) toutes facilités pour les collectes sur le terrain, y compris l'utilisation d'un hélicoptère, et la préparation des échantillons au cours de la mission. Nous exprimons notre gratitude à notre jeune collègue D Barret qui a apporté lors des récoltes à l'île de la Possession une aide précieuse et efficace. 
Nous remercions également très vivement $\mathrm{P}$ Tarroux, du département de biologie (URA 686) de l'École normale supérieure (Paris) qui a collaboré très étroitement à l'analyse statistique des données expérimentales et dont la compétence fut indispensable à la bonne conduite de cette étude.

\section{RÉFÉRENCES}

Bailly S (1982) Marquage à l'argent ammoniacal des chromosomes mitotiques et des noyaux quiescents de l'Urodèle $P$ waltlii. Can $J$ Genet Cytol 24, 541-548

Bailly S, Dreux P, Voisin JF (1990) Caryotypes de Curculionides (Insectes, Coléoptères) des îles du Sud de l'océan Indien. $C R$ Séances Acad Sci Paris 311, $75-80$

Barrion AA, Saxena RC, Jilani G (1988) Spermatic cells and chromosomes of Sitophilus oryzae (L) and Sitophilus zeamais (Mots) (Coleoptera, Curculionidae). Cytologia 53, 659-664

Carlson KA (1972) A method for identifying homogeneous classes. Multivar Behav Res $7,483-488$

Chown SL (1989) Habitat use and diet as biogeographic indicators for subantarctic Ectemnorrhinini (Coleoptera, Curculionidae). Antarct Sci I, 23-30

Chown SL, Scholtz CH (1989) Immature stages of Curculionidae (Coleoptera) from the sub-Antarctic Prince Edward Islands. J Entomol Soc S Afr 52, 253-275

Dey SK (1986) The Xyyp sex-determining mechanism in Alcides sp (Coleoptera, Curculionidae). Chromosome Inf Serv 40, 20-21

Dreux P, Voisin JF (1987) Les peuplements de Coléoptères des Terres subAntarctiques de l'océan Indien. Bull Soc Zool Fr 112, 455-470

Dreux P, Voisin JF (1989) Sur la systématique des genres de la sous-famille des Ectemnorrhininae (Coleoptera, Curculionidae). Nouv Rev Entomol (NS) 6, 111-118 Jeannel R (1940) Croisière du Bougainville aux îles australes françaises III. Coleoptères. Mém Mus Nat Hist Nat (NS) 14, 63-201

Levan A, Fredga K, Sandberg AA (1964) Nomenclature for centromeric position on chromosomes. Hereditas 52, 201-220

Meyer JA (1974) Constel : a fortran IV program for factor and cluster analysis of mixed data. Behav Res Methods Instrum \& Comput 6, 506

Postiglioni A, Da Silva A, Ponce de León R, De Vaio ES (1987) Three species of Heilipodus (Coleoptera, Curculionidae) with different karyotypes and sex chromosome systems. Genetica $75,213-218$

Smith SG (1950) The cyto-taxonomy of Coleoptera. Can Entomol 82, 58-68

Stevens NM (1906) Studies in spermatogenesis II. Carnegie Inst Wash Publ 36, 33-74

Takenouchi Y (1984) The chromosomes of 8 species of the Curculionidae (Coleoptera). J Hokkaido Univ Educ (sect II B) 34, 1-6

Virrki N, Sepúlveda JM (1990) Chromosomes of the Puerto Rican «vaquita», Diaprepes abbreviatus (L) (Curculionidae, Otiorrhynchinae, Phyllobiini). J Agric Univ Press 74, 121-132 